\title{
Trifunctional antibodies induce efficient antitumour activity with immune cells from head and neck squamous cell carcinoma patients after radio-chemotherapy treatment
}

\author{
Petra Schroeder • Carsten Lindemann • Kirsten Dettmar · Jürgen Brieger • Jan Gosepath • Benjamin Pogorzelski \\ Diane Seimetz $\cdot$ Judith Atz
}

Received: 18 November 2010 / Accepted: 23 March 2011

\begin{abstract}
Background Trifunctional antibodies, such as catumaxomab (anti-EpCAM $\times$ anti-CD3) and ertumaxomab (antiHER-2/neu $\times$ anti-CD3), transiently link immune effector cells to tumour cells, which results in cellular cytotoxicity towards the tumour cells. A functional immune system is therefore essential for effective anti-tumour activity. However, the commonly observed haematotoxicity of chemotherapeutics and radiation therapy may be associated with some degree of immunosuppression. Combining chemotherapy and trifunctional antibodies in cancer treatment requires understanding of the impact of chemotherapeutics on immune cell function and, thus, on the activity of trifunctional antibodies.

Methods The effect of chemotherapeutic treatment on trifunctional antibody-mediated anti-tumour activity was
\end{abstract}

P. Schroeder · C. Lindemann

EUFETS GmbH

Vollmersbachstraße 66

Idar-Oberstein, Germany

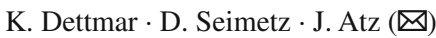

Fresenius Biotech $\mathrm{GmbH}$

Frankfurter Ring 193a

80807 Munich, Germany

e-mail: judith.atz@fresenius-biotech.com

J. Brieger

Department of Otolaryngology, Head and Neck Surgery

Medical Centre of the Johannes Gutenberg University Mainz

Mainz, Germany

J. Gosepath · B. Pogorzelski

Department of Otolaryngology

Dr. Horst Schmidt Kliniken GmbH

Ludwig-Erhard-Str. 100

Wiesbaden, Germany assessed in vitro. Blood samples were collected from 12 head and neck squamous cell carcinoma patients after chemotherapy (5-fluorouracil, cisplatin) and radiotherapy, and from one healthy control donor. The immune cell status was analysed and mononuclear cells (MNC) were isolated. The potency of catumaxomab and ertumaxomab was assessed in a cytotoxicity assay using MNC isolated from each patient sample in co-culture with a tumour target cell line. The release of inflammatory cytokines was also monitored in the cell culture supernatant.

Results Most patients included in this study had decreased immune cell counts during the course of chemotherapy. Nonetheless, an effective and concentration-dependent anti-tumour activity mediated by trifunctional antibodies was demonstrated using these patient immune effector cells. The immune response activity of the patient immune cells was not impaired one week after cisplatin administration or even three days after the last 5-fluorouracil treatment.

Conclusion This study shows for the first time that immune effector cells from cancer patients undergoing standard chemotherapy and radiotherapy can be activated by trifunctional antibodies for efficient killing of tumour cells.

Keywords Catumaxomab · Ertumaxomab · Trifunctional antibody $\cdot \mathrm{HNSCC} \cdot$ Chemotherapy $\cdot$ Immunotherapy

\section{Introduction}

Primary tumours are currently treated with a combination of different therapeutic strategies including surgery, local radiotherapy and chemotherapy. After the tumour appears to have been eradicated, micrometastases of dormant tumour cells may frequently lead to tumour relapse and therapeutic 
failure. To gain a lasting tumour remission, it is necessary to develop strategies to kill all cancer cells efficiently, by using an appropriate combination and schedule of chemotherapeutic agents, surgery and irradiation, and also to stimulate an immune response against residual tumour cells.

The idea of recruiting host immune effector cells to the tumour site by using therapeutic antibodies to provoke an anti-tumour effect was first proposed in the late 1980s. Initial studies of antibody-based cancer therapy used monospecific monoclonal antibodies. Monospecific antibodies bind specifically to one particular antigen expressed on tumour cells and evoke an anti-tumour response via several mechanisms including complement-dependent cytotoxicity, induction of apoptosis, inhibition of receptor dimerisation and downstream signalling, and many others [1, 2]. Bispecific antibodies, which are able to bind two different antigens, were developed to further enhance efficacy. Suitable target antigens for bispecific antibodies include tumourassociated antigens and an antigen expressed on immune effector cells such as $\mathrm{T}$ cells or natural killer (NK) cells [3-7], to enable a transient linkage between immune effector cells and tumour cells leading to immune cell activation and lysis of tumour cells. Trifunctional antibodies combine the characteristics of classical monospecific antibodies and bispecific molecules: In addition to the two specific antigen binding sites, trifunctional antibodies retain their intact Fc region, which mediates recruitment and activation of accessory cells (macrophages, dendritic cells, NK cells) [11]. Thus, the mode of action of these trifunctional antibodies is based on the simultaneous recruitment of $\mathrm{T}$ cells and accessory cells to the tumour site leading to improved tumour cell elimination by various immunologic killing mechanisms [8-13]. The trifunctional antibody catumaxomab (anti-EpCAM $\times$ anti-CD3, Removab ${ }^{\circledR}$ ) has been approved recently in the European Union for the intraperitoneal treatment of malignant ascites due to EpCAM-positive carcinoma where standard therapy is not available or no longer feasible $[8,9]$. The related trifunctional antibody ertumaxomab (anti-HER-2/neu $\times$ anti-CD3) is in clinical development.

Due to their unique mode of action, a functional immune system is essential for trifunctional antibodies to exert their full activity. Haematologic toxicity is a known side effect of chemotherapy and radiation therapy, and results in transient immunodeficiency. The resulting impairment of cellular and humoral immunity can last up to several months after the end of treatment [14-19]. Combining chemotherapy and trifunctional antibody-based immunotherapy in cancer treatment therefore requires evaluation of the immune response activity that remains after chemotherapy treatment.

The present study has therefore been conducted to provide first in vitro data in support of the treatment of cancer patients with trifunctional antibodies in combination with chemotherapy. Immune cells from head-and-neck squamous cell carcinoma (HNSCC) patients after cisplatin and 5-fluorouracil (5-FU) treatment were assessed for their remaining immune response activity. The functionality of these immune cells was evaluated by analysing the capac- ity of trifunctional antibodies (catumaxomab and ertumaxomab) to mediate cellular cytotoxicity towards tumour cells in vitro. Patient immune cells were taken at different time points before, during and after the chemotherapy cycles, and after radiation therapy in order to assess a potential impact on the immune response activity in relation to the time interval to the previous chemotherapy application or in relation to the number of previous treatment cycles (potential cumulative effects of several treatment cycles). 5-FU, a pyrimidine antagonist that inhibits DNA synthesis, is commonly used in the treatment of patients with neoplastic disorders, e.g., of the colon, head and neck, and breast [20-22]. Cisplatin, an alkylating agent that binds to DNA and interferes with its repair mechanisms [23], is used in the treatment of patients with, e.g., ovarian cancer, colon cancer and head and neck cancer. Promising results have been reported from combining monoclonal antibody treatment and chemotherapy treatment in cancer patients. For example, the combination of anti-EGFR monoclonal antibodies with chemotherapy increased the median survival in colorectal cancer patients [24] and the anti-HER-2/ neu antibody trastuzumab, combined with paclitaxel, was found to be an effective regimen as first-line chemotherapy in metastatic breast cancer [25].

This study included the analysis of each patient sample taken at different time points (before, during and after chemotherapy and after completion of radiation therapy, see Fig. 1 for details) for (i) characterisation of the status of immune cells, (ii) analysis of trifunctional antibody (catumaxomab and ertumaxomab)-mediated cellular cytotoxicity towards the EpCAM and HER-2/neu expressing SK-OV-3 human tumour cell line, and (iii) antibody-mediated release of cytokines (tumour necrosis factor alpha (TNF- $\alpha$ ), interferon gamma (IFN- $\gamma$ ), interleukin 2 (IL-2), IL-4, IL-5, IL-10).

\section{Patients and methods}

Study approval

The study was approved by the Ethics Committee of the Johannes Gutenberg University, Mainz, Germany (institutional review board no. 837.266.06, 5364). Informed consent was obtained from all patients prior to blood withdrawal.

Patient population and blood sampling

Whole blood samples from a total of 12 HNSCC patients aged 51-78 were provided by the Laboratory of Tumor Biology, Department of Otolaryngology, Johannes Gutenberg-University, Mainz, Germany. Patients received cisplatin and 5-fluorouracil (5-FU) following surgical intervention (Fig. 1). Cisplatin was administered intravenously 
A
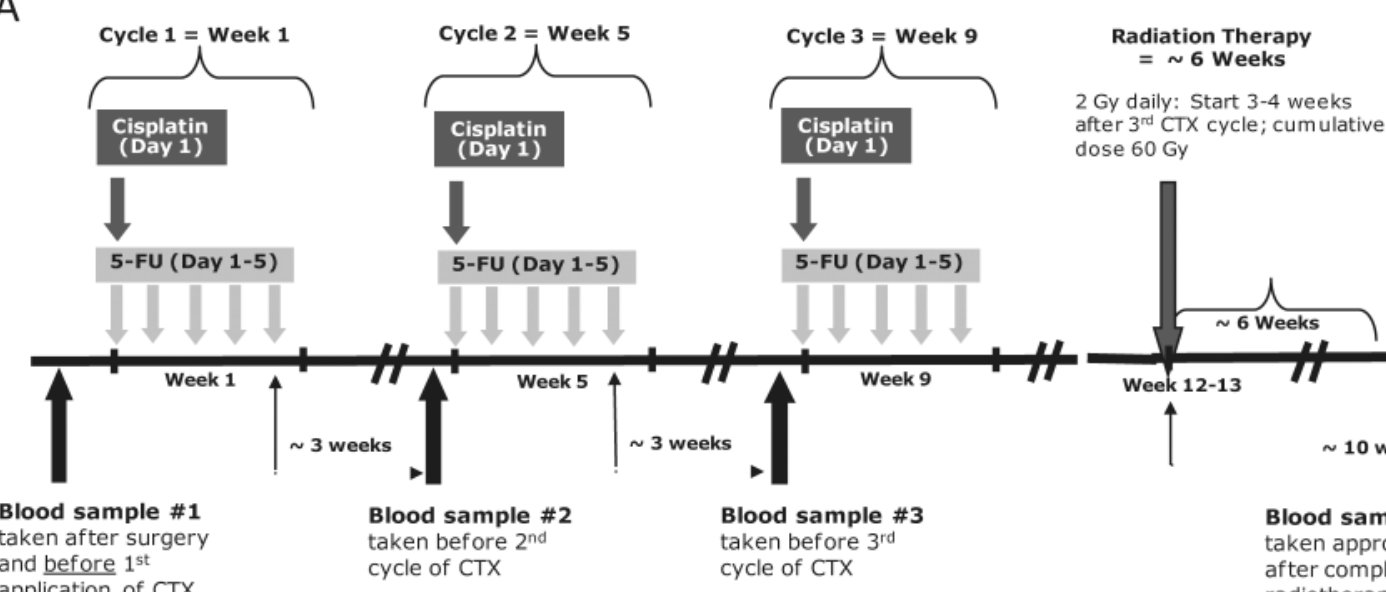

dose $60 \mathrm{~Gy}$

taken before $3^{\text {rd }}$

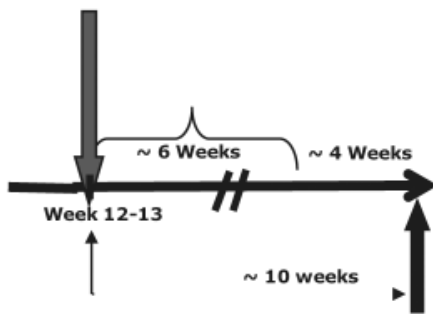

and before $1^{\text {st }}$

cycle of CTX

cycle of CTX

Blood sample \#4

taken approx. 4 weeks

after completion of radiotherapy

B
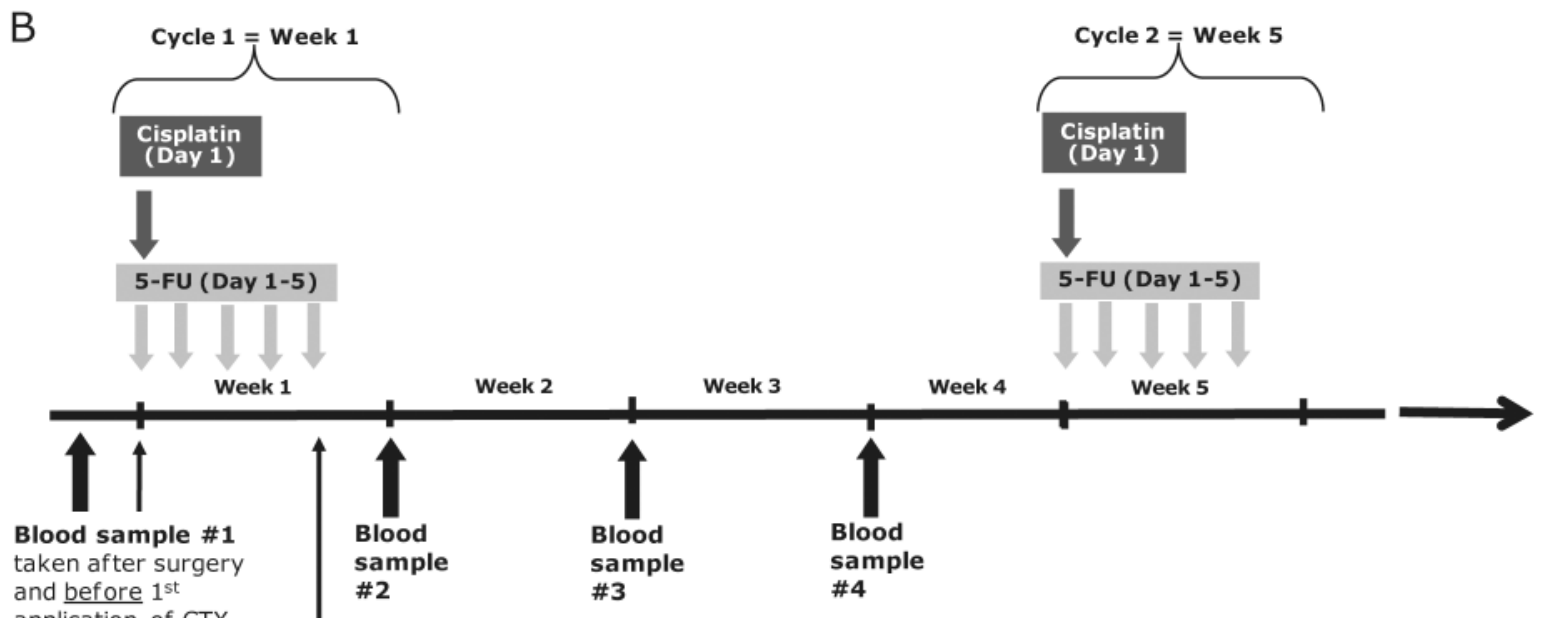

and before 1

application of CTX

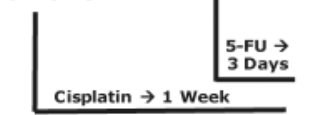

Time interval $\rightarrow$ Blood sample \#2

Fig. 1 (A) Scheme of monthly blood sampling of studies I (catumaxomab) and II (ertumaxomab) (9 patients included) (B) Scheme of weekly blood sampling of study III (ertumaxomab) (3 patients included)

at $100 \mathrm{mg} / \mathrm{m}^{2}$ body surface area on day 1 of the start of each 28-day treatment cycle. 5-FU was given intravenously at $1000 \mathrm{mg} / \mathrm{m}^{2}$ surface area on days $1-5$ of each cycle. Radiation therapy with 2 gray (Gy) daily was initiated three to four weeks after the third cycle of chemotherapy up to a cumulative dose of $60 \mathrm{~Gy}$.

The in vitro study consisted of three parts: Studies I (catumaxomab; monthly sampling) and II (ertumaxomab; monthly sampling) comprised the same 9 patients, and study III (ertumaxomab; weekly sampling) comprised 3 additional patients. A total of four blood samples were taken from each patient and analysed within $24 \mathrm{~h}$ after blood collection.
In all three study parts, the first blood sample was taken following surgery and before the first application of chemotherapy. In studies I and II, the subsequent two blood samples were taken at monthly intervals immediately before application of the next chemotherapy cycle, about three weeks after the previous 5-FU application (Fig. 1A). The fourth and last blood sample was taken four weeks after completion of the radiation therapy (approximately 6 weeks duration) that was performed after the third cycle of chemotherapy.

In study III, blood sampling was done weekly for 3 weeks (until one week before application of the second cisplatin and 5-FU cycle). The shortest time interval in 
this schedule was assessed with sample \#2 taken one week after the cisplatin administration on day 1 and three days after the last 5-FU dose on day 5 (Fig. 1B). As controls, blood samples from one healthy donor were drawn every two weeks, up to a total of three samples, and analysed in parallel to and in the same way as the patient samples.

All experimental analyses were performed at EUFETS $\mathrm{GmbH}$, Idar-Oberstein, Germany, on behalf of Fresenius Biotech GmbH, Munich, Germany.

\section{Staining of immune cells}

Each blood sample was analysed for total cell counts and cellular composition. Leukocyte subsets of interest were analysed by flow cytometry. White blood cells were stained with specific fluorochrome-conjugated antibodies specific for CD3 (FITC, clone UCHT1, Beckman Coulter, Krefeld, Germany; Tritest T-cells, Becton Dickinson, Heidelberg, Germany) expressed on T cells, CD4 (PE, clone 13B8.2, Beckman Coulter; Tritest T-cells) on T-helper cells, CD8 (PE, clone B9.11, Beckman Coulter; Tritest T-cells) on cytotoxic T-cells, CD19 (PE, clone J4.119, Beckman Coulter, Tritest B-cells, Becton Dickinson) on B cells, CD16/CD56 (PE, clone 3G8+N901, Beckman Coulter; Tritest NK cells, Becton Dickinson) on NK cells, and CD14 (PE, clone RMO52, Beckman Coulter; SimultTest LeucoGATE, Becton Dickinson) on monocytes. Cells were gated by CD45staining (FITC, clone J.33, Beckman Coulter; SimultTest LeucoGATE) to exclude dead cells and debris. T cells were defined as $\mathrm{CD} 3+$, cytotoxic T-cells as $\mathrm{CD} 8+/ \mathrm{CD} 3+$, T-helper cells as CD4+/CD3+, B cells as CD19+/CD3-, NK cells as $\mathrm{CD} 16+\mathrm{CD} 56+/ \mathrm{CD} 3-$ and monocytes as CD14+. Expo 32 software (Beckmann Coulter) or CellQuest Pro software (Becton Dickinson) were used for data acquisition and analysis of immune status. The leukocyte subsets (positive cells for each antigen) were expressed as a percentage of the total nucleated cell count.

\section{Cytotoxicity assay}

Mononuclear cells (MNC) from patients or from a healthy donor (control blood samples) were isolated by density gradient centrifugation following a 1:2 dilution with PBS (Invitrogen) using lymphocyte separation medium (PAA).

The SK-OV-3 tumour target cell line (human ovarian tumour; EpCAM and HER-2/neu-positive) was purchased from ATCC (LGC Promochem, Wesel, Germany) and a primary and secondary cell bank was established. Cells were used within 15 passages resulting in a maximum culture period of 12 weeks. The cells were cultured in McCoy's 5A medium with $2 \mathrm{mM}$ Glutamax (Invitrogen, Karlsruhe, Germany) and $10 \%$ foetal bovine serum (FBS; PAA, Pasching, Austria). SK-OV-3 cells were seeded $\left(1 \times 10^{5}\right.$ cells $\left./ \mathrm{ml}\right)$ into 96-well microtitre plates and allowed to adhere overnight at $37^{\circ} \mathrm{C}, 5 \% \mathrm{CO}_{2}$ in a humidified atmosphere.
MNC were added to the tumour cell cultures at an effector to target ratio of 10:1, in RPMI-1640 culture medium (Invitrogen) supplemented with 10\% FBS (PAA) and 2\% penicillin and streptomycin (Invitrogen), and co-cultured in the presence of various concentrations of trifunctional antibodies (catumaxomab or ertumaxomab: 17.6 to 0.069 $\mathrm{ng} / \mathrm{ml}$ in serial 2-fold dilutions) or without antibodies as control. Catumaxomab and ertumaxomab were provided by Fresenius Biotech (Munich, Germany). Each antibody concentration was assayed in replicates of 4-8, depending on the number of patient MNC available. Co-cultures of MNC and SK-OV-3 tumour cells were incubated for 3-4 days at $37^{\circ} \mathrm{C}, 5 \% \mathrm{CO}_{2}$ in a humidified atmosphere. After collection of cell-free supernatants for cytokine quantification, the immune effector cells (MNC) were removed by extensive washing with PBS. The residual tumour cells were quantified with the XTT cell proliferation kit II (Roche, Penzberg, Germany), according to the manufacturer's instructions. Absorbance was measured at a wavelength of 450 $\mathrm{nm}$ using a microplate reader (Spectrafluor Plus; Tecan, Crailsheim, Germany) and the software Magellan (Tecan) was used for data acquisition. Cytotoxicity in vitro was determined by calculating the percentage of residual tumour cells as follows:

residual tumour cells $(\%)=\frac{\text { mean absorbance sample }- \text { mean absorbance medium control }}{\text { mean absorbance control without antibody }- \text { mean absorbance medium control }} \times 100$

Tumour cell killing of $>80 \%$ (residual tumour cells $<20 \%$ ) was defined as efficient killing, indicating functional activity of the patient MNC.

Quantification of cytokines in co-culture supernatants

Release of the cytokines TNF- $\alpha$, IFN- $\gamma$, IL-2, IL-4, IL-5 and IL-10 was quantified in cell-free supernatants from co-cultures after the end of the incubation period (ertumaxomab studies II and III, only). Samples were stored at $-80^{\circ} \mathrm{C}$ until analysis. Cytokines were quantified by cytometric bead array (CBA; Becton Dickinson), according to the manufacturer's instructions. Briefly, six bead populations with distinct fluorescence intensities were coated with cytokine-specific murine capture antibodies. The bead populations were mixed and incubated with recombinant cytokine standards or test samples and PE-conjugated antimouse antibodies. Acquisition and analysis of sample data was performed by flow cytometry and the manufacturer's CBA analysis software (Becton Dickinson).

Deviations to blood sampling schedule

In deviation to the proposed sampling scheme shown in Fig. 1A (monthly sampling), the second blood sample of two (out of nine) patients was taken immediately after the application of cisplatin. Therefore, results obtained from these two samples were not used in any calculation. 


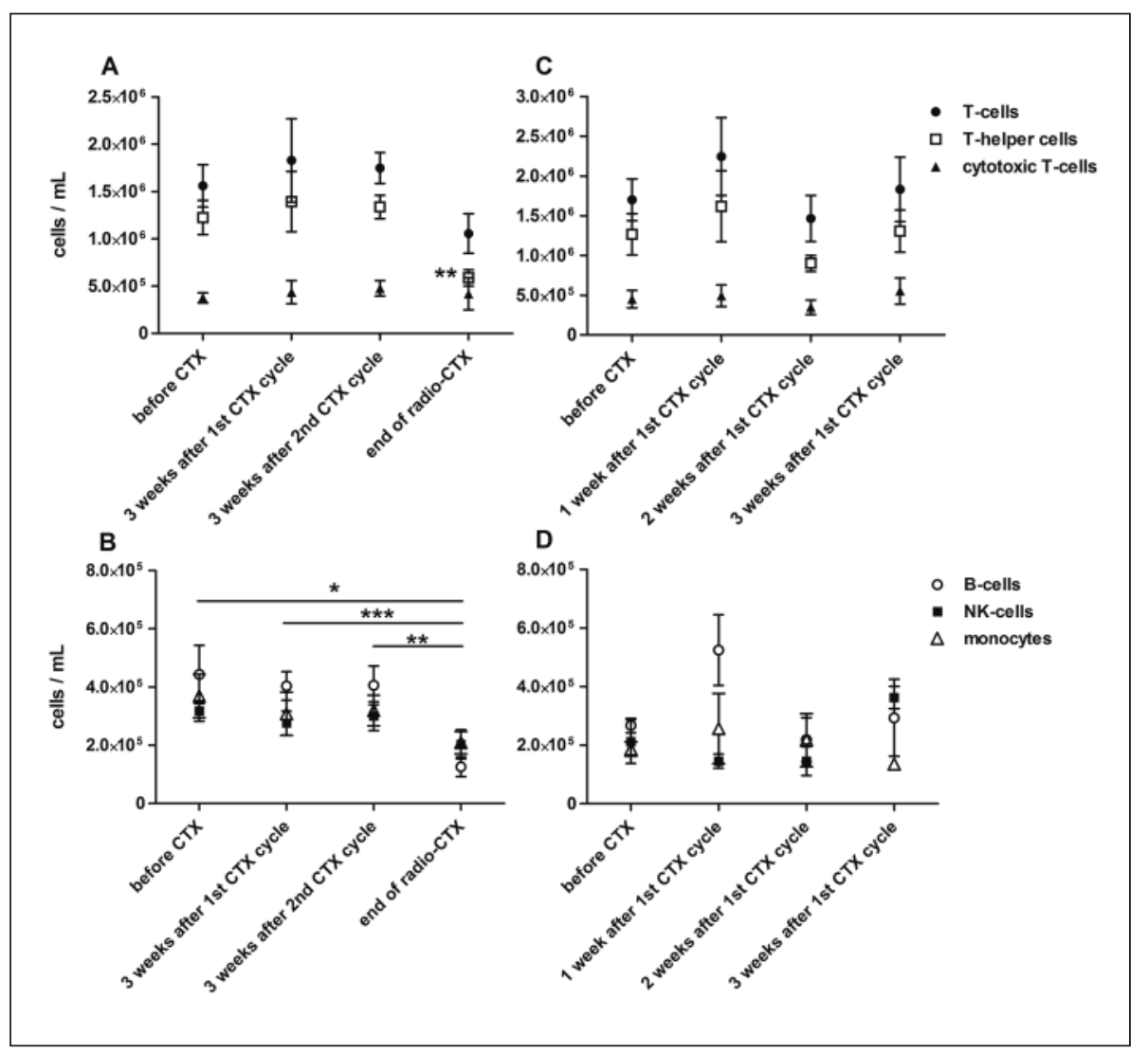

Fig. 2 Counts of immune cells from HNSCC patients (A) T cells and T-cell subtypes of studies I (catumaxomab, monthly interval) and II (ertumaxomab, monthly interval) (B) B cells, NK cells and monocytes of studies I and II (C) T cells and T-cell subtypes of study III (ertumaxomab, weekly interval) (D) B cells, NK cells and monocytes of study III. $* * * p=0.0004$ (extremely significant), $* * p<0.0085$ (very significant), ${ }^{*} p=0.01$ (significant)

Statistics

GraphPad Prism software (version 5.0.2; GraphPad Software, Inc., La Jolla, CA, USA) was used for all statistical analyses. The cytotoxicity results were analysed using a non-linear fit of normalised data (as indicated in the GraphPad Prism software) and the $\log E_{50}$ (logarithmic effective concentration 50; the concentration that provokes a response halfway between the baseline and maximum response) was calculated. Any significant differences in $\log \mathrm{EC}_{50}$ values were determined by one-way analysis of variance (ANOVA) and any significant differences in immune cell counts were calculated using the unpaired Student's $t$-test $(p=0.01-0.05$ significant $* ; p=0.001-0.01$ very significant ${ }^{* *} ; p<0.001$ extremely significant $\left.* * *\right)$.

\section{Results}

Characterisation of immune cells from HNSCC patient samples

All patient samples were analysed for lymphocyte subsets at different time points before, during and after the chemotherapeutic treatment course in order to relate immune cell composition to the results obtained in the functional assay (cytotoxicity assay). The total numbers of $\mathrm{T}$ cells, T-helper cells and cytotoxic T-cells of patient samples $(n=9)$ from the monthly sampling schedule are presented in Fig. 2A. Total counts of B cells as well as NK cells and monocytes from these samples are shown in Fig. 2B. Compared to the pre-treatment samples, a slight increase in $\mathrm{T}$ cells was observed three weeks after the first and second courses of chemotherapy (CTX; before the second and third CTX cycles, respectively). At the end of the treatment period (after three CTX cycles and six weeks of radiotherapy) there was a significant decrease in T-helper cell counts compared to pre-treatment samples $(p=0.008)$. At the end of radiochemotherapy, an inverse ratio of T-helper and cytotoxic T-cells was observed in two patient samples $(<1: 1$; lower counts of T-helper than cytotoxic T-cells). In all other samples, the ratio of T-helper and cytotoxic T-cells was physiological; i.e., the T-helper cell count was higher than that of cytotoxic T-cells (>1:1). However, the abnormal ratio of T-helper and cytotoxic T-cells in the two samples did not affect antibody-mediated cytotoxicity of the trifunctional antibodies.

In study III blood samples from three HNSCC cancer patients were collected in weekly intervals up to three weeks following the first cycle of cisplatin and 5-FU. As depicted in Fig. 2C and D, the first application of 5-FU and cisplatin did not affect T-cell counts, T-cell subsets, NK cell counts or monocyte counts. However, B-cell counts were slightly increased one week after the first cycle of CTX. There were no changes in immune cell subsets in three 


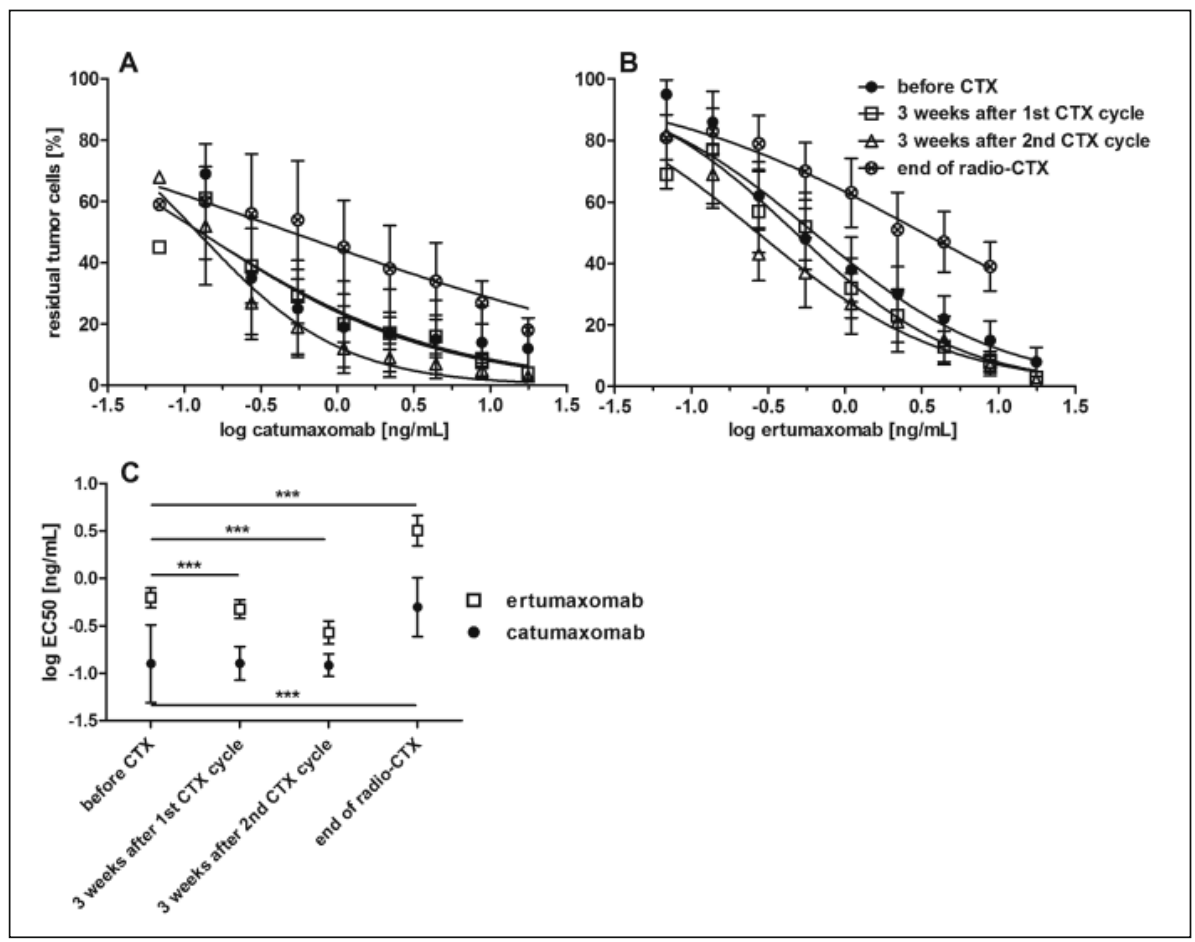

Fig. 3 Catumaxomab- (A) and ertumaxomab- (B) mediated cytotoxicity of MNC from HNSCC patients before, at several monthly time-points during, and at the end of radio-chemotherapy (studies I and II). ( $\bullet$ : samples obtained before start of CTX, $\square$ : samples from 3 weeks after the first and immediately before the second cycle of CTX; $\Delta$ : samples obtained 3 weeks after the second and immediately before the third cycle of CTX; $\otimes$ : samples obtained after the fourth cycle of CTX and after a cumulative radiation dose of $60 \mathrm{~Gy}$ ) (C) Concentration of catumaxomab $(\bullet$, study I) and ertumaxomab ( $\square$, study II) for killing $50 \%$ of the tumour target cells (EC50) before, during and after the end of radiochemotherapy. $* * * p<0.0001$ (extremely significant)

blood samples taken every two weeks from one healthy donor (data not shown).

Trifunctional antibodies mediate efficient tumour cell killing using immune cells from cancer patients after cisplatin and 5-FU treatment

Patient immune cells obtained at monthly intervals in the context of chemotherapy treatment induced efficient antitumour activity by the trifunctional antibodies catumaxomab and ertumaxomab; activity was comparable to the pre-treatment samples (immune cells before first cycle). Catumaxomab-mediated tumour cell killing by patient immune effector cells is shown in Fig. 3A (study I, catumaxomab) and results obtained with ertumaxomab are presented in Fig. 3B (study II, ertumaxomab). Both trifunctional antibodies mediated dose-dependent cytotoxicity towards human SK-OV-3 tumour cells in the presence of patient immune cells taken at monthly intervals. Immune cells obtained three weeks after the first and second cycles of cisplatin and 5-FU resulted in efficient, concentrationdependent tumour cell killing in both studies $(>80 \%)$. The dose-dependent killing was maintained even after a third cycle of chemotherapy and a cumulative radiation dose of $60 \mathrm{~Gy}$. Although the number of T cells was strongly reduced in all patient samples obtained at the end of radiochemotherapy, the remaining immune effector cell function was still potent enough to result in efficient cytotoxicity mediated by trifunctional antibodies.

As controls, three blood samples from one healthy donor taken at 2-weekly intervals were analysed in cytotoxic- ity assays using ertumaxomab. All three control samples showed comparable antibody concentration-dependent cytotoxicity ( $\log \mathrm{EC}_{50}:-0.24 \pm 0.24 \mathrm{ng} / \mathrm{ml} ; n=3$ ).

The results further indicate that the patient immune cells may become more susceptible to activation by trifunctional antibodies for efficient tumour cell killing during treatment with cisplatin and 5-FU. In Fig. 3C, the log EC50 values of studies I (catumaxomab; monthly sampling) and II (ertumaxomab; monthly sampling) are shown. The log $\mathrm{EC}_{50}$ values for ertumaxomab were significantly reduced when comparing patient immune cells taken three weeks after the first cycle (study II) vs. log $\mathrm{EC}_{50}$ values obtained with immune cells from patients before chemotherapy $\left({ }^{*} p<0.0001\right)$. Following the second cycle of chemotherapy the $\log \mathrm{EC}_{50}$ of ertumaxomab was even further reduced to a significant level $\left({ }^{*} p<0.0001\right)$ compared to pre-treatment. Experiments with catumaxomab show that the antibody concentration required for the killing of 50\% of SK-OV-3 tumour cells was similar in samples obtained before the treatment and three weeks after the first and second cycle of cisplatin and 5-FU, respectively. However, after the third chemotherapy cycle and radiation therapy the $\log \mathrm{EC}_{50}$ was significantly increased in both studies when compared to the pre-treatment $\log \mathrm{EC}_{50}$ values (I, catumaxomab and II, ertumaxomab; $p<0.0001$ ), indicating that radiotherapy mediates a stronger immunosuppressive effect than chemotherapy under these conditions.

Impaired immune cell function due to chemotherapy treatment was observed for the patient immune cells taken immediately after the intravenous infusion of cisplatin (two samples). There was no antibody-dependent specific anti-tumour activity with catumaxomab or ertumaxomab 


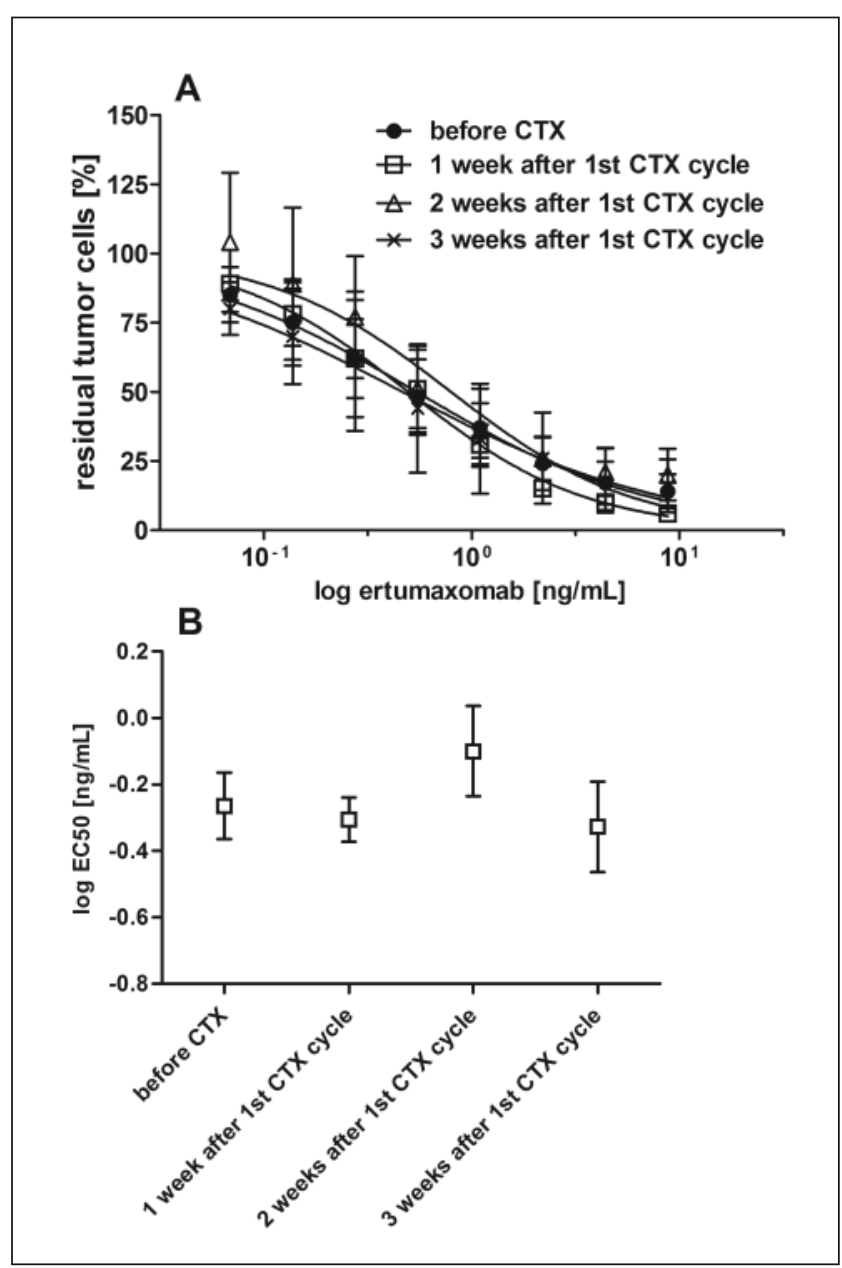

Fig. 4 (A) Ertumaxomab-mediated cytotoxicity of MNC from HNSCC patients obtained before, and at weekly intervals between the first and second cycles of chemotherapy (study III). ( $\bullet$ : samples obtained before start of CTX, $\square$ : samples from 1 week after the first cycle of CTX; $\Delta$ : samples obtained 2 weeks after the first cycle of CTX; $\times$ : samples obtained 3 weeks after the first cycle of CTX) (B) Concentration of ertumaxomab (study III) for killing 50\% of the tumour target cells (EC50) before and at weekly intervals between the first and second cycles of chemotherapy

against the SK-OV-3 tumour target cells in the presence of MNC isolated from these samples (data not shown).

In summary, immune cells taken three weeks after the respective previous chemotherapy cycle (monthly sampling schedule) mediated effective anti-tumour activity in vitro induced by the trifunctional antibodies catumaxomab and ertumaxomab, indicating a potent immune response activity of the patient cells that remains even after several cycles of chemotherapeutic treatment.

We were then interested to further evaluate the effect of a single cycle of cisplatin and 5-FU, and to investigate a time interval closer to the previous chemotherapy $(<$ three weeks). For this purpose, blood samples were taken weekly between the first and second cycles of chemotherapy (study III, ertumaxomab; Fig. 1B). With all samples from the weekly sampling schedule, concentration-dependent antibody-mediated killing of tumour target cells was found (Fig. 4A). Furthermore, there were no differences in tumour cell killing activity $\left(\log \mathrm{EC}_{50}\right)$ between the different sampling time points (Fig. 4B). This finding, observed also for sample 2 ( 1 week after first CTX cycle), indicates that even one week after the cisplatin administration (day 1) and as soon as three days after the last 5-FU dose (day 5), there was no functional impairment of the immune cells of these cancer patients due to the previous chemotherapeutic treatment.

Trifunctional antibodies induce the release of inflammatory cytokines in vitro

Cell-free supernatants were collected from the cytotoxicity assays from studies II (ertumaxomab; monthly sampling schedule) and III (ertumaxomab; weekly sampling schedule) and cytokines were quantified using a flow cytometric bead array. Ertumaxomab mediated concentration-dependent release of the inflammatory cytokines IFN- $\gamma$ (Fig. 5A), TNF- $\alpha$ (Fig. 5B), IL-5 (Fig. 5C) and IL-10 (Fig. 5D) in all patient samples taken at monthly intervals (study II). However, antibody-induced release of IL-2 and IL-4 was minor in the majority of samples (data not shown). Higher IFN- $\gamma$ levels were found in blood samples obtained during chemotherapy (three weeks after the first and second cycles of chemotherapy) compared to the levels obtained before any chemotherapeutic treatment. Additionally, high levels of TNF- $\alpha$ were observed and increased in samples obtained at the end of the radio-chemotherapy.

Cytokine levels in samples from study III (weekly sampling schedule) increased with increasing ertumaxomab concentrations in the in vitro assay. However, in this weekly sampling interval, no significant changes in cytokine levels were observed over time (data not shown). The control sample using MNC from a healthy donor also showed an increase in IFN- $\gamma$, TNF- $\alpha$, IL-10 and IL- 5 dependent on the antibody concentration used in vitro; lower levels of IL-4 and IL-2 were detected (data not shown).

\section{Discussion}

Standard chemotherapy and radiation therapy may have inhibitory effects on immune cells. For example, cisplatin and 5-FU are known to induce lymphocytopenia, resulting in transient immunosuppression in cancer patients. These drugs are also reported to enhance or permit efficient tumour cell killing in vitro at concentrations comparable with the maximally achieved therapeutic concentration in vivo in humans [26]. There is recent evidence indicating that some cytotoxic compounds and radiotherapy promote specific anticancer immune responses that contribute to the overall therapeutic effect $[27,28]$. This study investigated whether trifunctional antibodies are able to induce efficient 

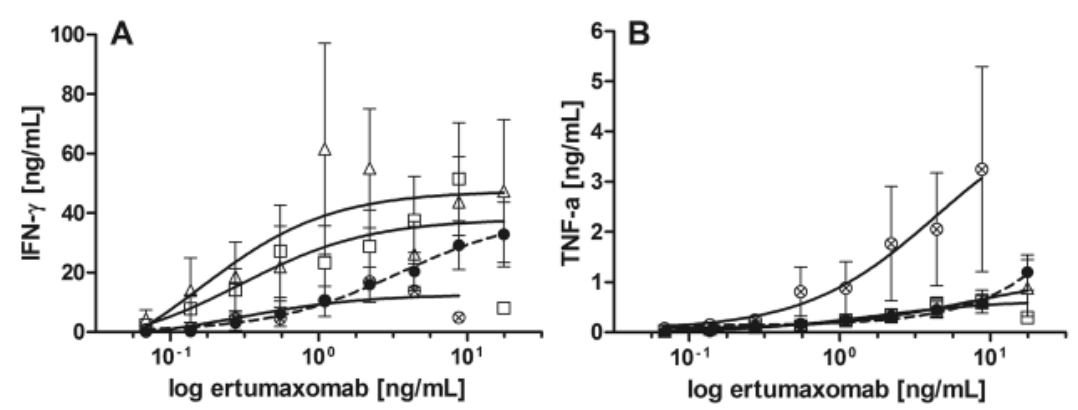

Fig. 5 Release of IFN- $\gamma$, TNF- $\alpha$, IL-5 and IL-10 in response to increasing ertumaxomab concentrations (study II; ertumax-

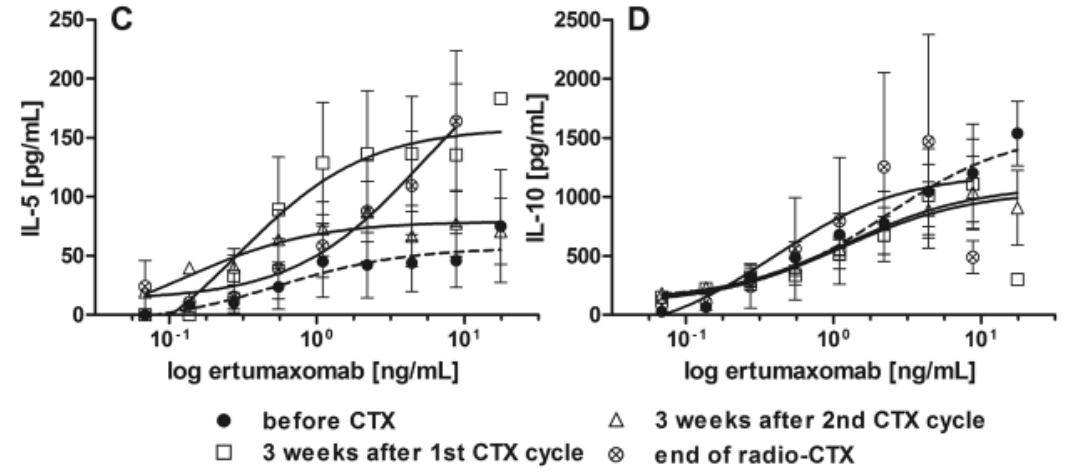
omab, monthly interval, 8 patients included; •, before CTX; $\square, 3$ weeks after first CTX cycle; $\Delta, 3$ weeks after second CTX cycle; $\otimes$, end of radio CTX)

anti-tumour activity with immune effector cells obtained from patients with HNSCC before, during and after chemotherapy, and following radio-chemotherapy.

In our study, most patients showed a decrease in $\mathrm{T}$ cell, B cell, NK cell and monocyte counts during the course of chemotherapy treatment, suggesting a suppressive effect of chemotherapy on the immune system. However, regardless of the chemotherapeutic treatment cycle, both trifunctional antibodies mediated efficient concentration-dependent antitumour activity with immune cells from these patients. Even after three previous chemotherapeutic cycles of cisplatin (cumulative dose $300 \mathrm{mg} / \mathrm{m}^{2}$ ) and 5-FU (cumulative dose $15,000 \mathrm{mg} / \mathrm{m}^{2}$ ) and a cumulative radiation dose of $60 \mathrm{~Gy}$, the patient immune effector cells retained their potential for efficient antibody-mediated tumour cell killing. Moreover, the killing efficacy of ertumaxomab was comparable using either effector cells from a healthy donor or from chemotherapy patients. Notably, using effector cells obtained three weeks after the first and second courses of cisplatin and 5-FU administration, antibody-mediated antitumour activity in vitro was enhanced (lower EC50) compared to pre-treatment, indicating that the patient immune cells may become even more susceptible to the activation by trifunctional antibodies. Our results are supported by the in vitro data of $\mathrm{Wu}$ and colleagues [29], who showed that the percentage of T-regulatory cells and IFN- $\gamma$ secreting CD8-positive $\mathrm{T}$ cells decreased remarkably during the course of chemotherapy when blood samples were taken from ovarian cancer patients at different time points during paclitaxel and carboplatin chemotherapy. Thus, chemother- apy may induce temporary immune reconstitution and may augment an anti-tumour immune response. Radiotherapy may also contribute to enhance killing activity of immune cells since irradiation mediates many effects on cells and tissues that may stimulate an immune response [30]. For example, low doses of ionising irradiation upregulate expression of MHC class I molecules [31] and tumourassociated antigens [32], as well as adhesion molecules by endothelial cells [33], thereby boosting cytotoxic T-cell activity [34].

Data from our study showed that even one week after cisplatin administration (day 1) and as soon as three days after the last 5-FU dose (day 5), trifunctional antibodies are able to activate immune effector cells of cancer patients for efficient tumour cell killing, indicating that the chemotherapeutic substances did not cause any relevant functional loss of the immune effector cells. Effector function was only impaired in two samples taken immediately after the intravenous infusion of cisplatin. Nonetheless, the observed lack of antibody-dependent specific cytotoxicity of these two samples demonstrates the suitability of this in vitro assay for testing the functionality of patient immune effector cells.

Inflammatory cytokine release is part of the mode of action of the trifunctional antibodies catumaxomab and ertumaxomab [10]. In a phase I trial of ertumaxomab in metastatic breast cancer, measurements of cytokines (IL-6, IL-2, TNF- $\alpha$ and IFN- $\gamma$ ) suggest a strong T-helper cell type 1-associated immune response [35]. In addition, a randomised phase II/III trial of catumaxomab in malig- 
nant ascites has demonstrated that cytokine-release-related symptoms may be positively correlated with efficacy and may serve as a predictive factor for the efficacy of catumaxomab [8, 36]. IFN- $\gamma$ is an inflammatory cytokine released from activated T cells and NK cells, and is known to exert anti-proliferative effects on neoplastic cells [37]. In our in vitro assays IFN- $\gamma$ levels were increased using patient blood samples taken three weeks after the first and second cycles of chemotherapy correlating to the increased cytotoxic activity mediated by these immune effector cells. Based on this observation, trifunctional antibody-induced release of IFN- $\gamma$ may be further enhanced in combination with cisplatin therapy. The efficient anti-tumour activity in parallel with the enhanced release of anti-neoplastic, pro-inflammatory cytokines such as IFN- $\gamma$ and TNF- $\alpha$ in our assays may suggest synergistic effects of the combination of trifunctional antibody and chemotherapy. Further supportive results for the combination of chemotherapeutics and trifunctional antibodies have been obtained in other preclinical models. In two ex vivo studies using the chicken embryo chorioallantoic membrane assay, the combination of catumaxomab and cisplatin enhanced the antitumour activity of immune effector cells from squamous cell carcinoma patients against autologous tumour cells $[38,39]$. Notably, the effect of each individual drug alone was lower. Furthermore, in a multicellular tumour spheroid model, combined administration of catumaxomab and cisplatin resulted in a synergistic anti-tumour effect with a strong reduction of tumour spheroid volume [40]. These reports indicate synergy for the combination of trifunctional antibodies with different chemotherapeutic drugs. In our study, no negative influence of the alkylating agent cisplatin and the pyrimidine antagonist 5-FU was observed on the trifunctional mode of action in vitro using immune cells from patients undergoing chemotherapy. Catumaxomab and ertumaxomab are able to mediate effective killing of tumour cells by immune cells from patients treated with chemotherapy. The patient immune cells can be activated by these bispecific antibodies already one week after chemotherapy with cisplatin and already three days after 5-FU treatment, resulting in significant tumour cell killing in vitro. Furthermore, our preliminary results indicate that the patient immune cells may become even more susceptible to activation by trifunctional antibodies for efficient tumour cell killing during the course of chemotherapy treatment.

These first results provide a preclinical starting point for combining chemotherapeutic drugs with trifunctional antibodies in the clinical setting. Thus, the appropriate selection of a chemotherapeutic drug or drug combination in an appropriate sequence could further enhance the anti-tumour activity of trifunctional antibodies in cancer patients.

Acknowledgements We thank the patients of the Department of Otolaryngology, Head and Neck Surgery, University, Medical Centre of the Johannes Gutenberg University Mainz, Mainz, Germany and the laboratory staff of EUFETS GmbH.

Conflict of interest The authors declare that they have no conflict of interest relating to the publication of this manuscript.

\section{References}

1. Köninki K, Barok M, Tanner M et al (2010) Multiple molecular mechanisms underlying trastuzumab and lapatinib resistance in JIMT-1 breast cancer cells. Cancer Lett 294:211-219

2. Ciavarella S, Milano A, Dammacco F, Silvestris F (2010) Targeted therapies in cancer. BioDrugs 24:77-88

3. Gall JM, Davol PA, Grabert RC et al (2005) T cells armed with anti-CD3 $\mathrm{x}$ anti-CD20 bispecific antibody enhance killing of CD20+ malignant B cells and bypass complement-mediated rituximab resistance in vitro. Exp Hematol 33:452-459

4. Watanabe M, Wallace PK, Keler T et al (1999) Antibody dependent cellular phagocytosis (AD$\mathrm{CP})$ and antibody dependent cellular cytotoxicity (ADCC) of breast cancer cells mediated by bispecific antibody, MDX-210. Breast Cancer Res Treat 53:199-207

5. Wimberger P, Xiang W, Mayr D et al (2003) Efficient tumour cell lysis by autologous, tumourresident $\mathrm{T}$ lymphocytes in primary ovarian cancer samples by an EP-CAM-/CD3-bispecific antibody. Int J Cancer 105:241-248

6. Chan JK, Hamilton CA, Cheung MK et al (2006) Enhanced killing of primary ovarian cancer by retargeting autologous cytokine-induced killer cells with bispecific antibodies: a preclinical study. Clin Cancer Res 12:1859-1867

7. Reusch U, Sundaram M, Davol PA et al (2006) Anti-CD3 $x$ anti-epidermal growth factor receptor (EGFR) bispecific antibody redirects T-cell cytolytic activity to EGFR-positive cancers in vitro and in an animal model. Clin Cancer Res 12:183-190

8. Seimetz D, Lindhofer H, Bokemeyer C (2010) Development and approval of the trifunctional antibody catumaxomab (anti-EpCAMxanti-CD3) as a targeted cancer immunotherapy. Cancer Treat Rev 36:458-467

9. Heiss MM, Murawa P, Koralewski P et al (2010) The trifunctional antibody catumaxomab for the treatment of malignant ascites due to epithelial cancer: results of a prospective randomized phase II/III trial. Int J Cancer 127:2209-2221

10. Zeidler R, Reisbach G, Wollenberg B et al (1999) Simultaneous activation of T-cells and accessory cells by a new class of intact bi-specific antibody results in efficient tumor cell killing. J Immunol 163:1246-1252

11. Zeidler R, Mysliwietz J, Csanady M et al (2000) The Fc-region of a new class of intact bi-specific antibody mediates activation of accessory cells and NK cells and induces direct phagocytosis of tumor cells. Br J Cancer 83:261-266

12. Zeidler R, Mayer A, Gires O et al (2001) TNFalpha contributes to the anti-tumor activity of a bispecific, trifunctional antibody. Anticancer Res 21:3499-3503

13. Ruf P, Gires O, Jäger M et al (2007) Characterisation of the new EpCAM-specific antibody HO-3: implications for trifunctional antibody immunotherapy of cancer. Br J Cancer 97:315-321

14. Alanko S, Pelliniemi TT, Salmi TT (1992) Recovery of blood B-lymphocytes and serum immunoglobulins after chemotherapy for childhood acute lymphoblastic leukemia. Cancer 69:1481-1486

15. Campbell MJ, Scott J, Maecker HT (2005) Immune dysfunction and micrometastases in women with breast cancer. Breast Cancer Res Treat 91:163-171

16. Zignol M, Peracchi M, Tridello G et al (2004) Assessment of humoral immunity to poliomyelitis, tetanus, hepatitis B, measles, rubella, and mumps in children after chemotherapy. Cancer 101:635-641
17. Robert F, Childs HA, Spencer SA et al (1999) Phase I/IIa study of concurrent paclitaxel and cisplatin with radiation therapy in locally advanced non-small cell lung cancer: analysis of early and late pulmonary morbidity. Semin Radiat Oncol 9:136-147

18. Alanko S, Salmi TT, Pelliniemi TT (1994) Recovery of blood T-cell subsets after chemotherapy for childhood acute lymphoblastic leukemia. Pediatr Hematol Oncol 11:281-292

19. Arrieta O, Michel Ortega RM, VillanuevaRodríguez G et al (2010) Association of nutritional status and serum albumin levels with development of toxicity in patients with advanced non-small cell lung cancer treated with paclitaxelcisplatin chemotherapy: a prospective study. BMC Cancer 10:50

20. Standish LJ, Torkelson C, Hamill FA et al (2008) Immune defects in breast cancer patients after radiotherapy. J Soc Integr Oncol 6:110-121

21. Peters GJ, van Triest B, Backus HH et al (2000) Molecular downstream events and induction of thymidylate synthase in mutant and wild-type p53 colon cancer cell lines after treatment with 5-fluorouracil and the thymidylate synthase inhibitor raltitrexed. Eur J Cancer 36:916-924

22. Shah MA, Schwartz GK (2001) Cell cycle-mediated drug resistance: an emerging concept in cancer therapy. Clin Cancer Res 7:2168-2181

23. Jamieson ER, Lippard SJ (1999) Structure, recognition, and processing of cisplatin-DNA adducts. Chem Rev 99:2467-2498

24. Saridaki Z, Georgoulias V, Souglakos J (2010) Mechanisms of resistance to anti-EGFR monoclonal antibody treatment in metastatic colorectal cancer. World J Gastroenterol 16:1177-1187

25. Han HS, Kim JS, Park JH et al (2009) Weekly paclitaxel and trastuzumab as a first-line therapy 
in patients with HER2-overexpressing metastatic breast cancer: magnitude of HER2/neu amplification as a predictive factor for efficacy. J Korean Med Sci 24:910-917

26. Markasz L, Skribek H, Uhlin M et al (2008) Effect of frequently used chemotherapeutic drugs on cytotoxic activity of human cytotoxic T-lymphocytes. J Immunother 31:283-293

27. Zitvogel L, Apetoh L, Ghiringhelli F, Kroemer G (2008) Immunological aspects of cancer chemotherapy. Nat Rev Immunol 8:59-73

28. Zitvogel L, Apetoh L, Ghiringhelli F et al (2008) The anticancer immune response: indispensable for therapeutic success? J Clin Invest 118:19912001. Review

29. Wu X, Feng QM, Wang Y et al (2010) The immunologic aspects in advanced ovarian cancer patients treated with paclitaxel and carboplatin chemotherapy. Cancer Immunol Immunother 59:279-291

30. Friedman EJ (2002) Immune modulation by ionizing radiation and its implications for cancer immunotherapy. Curr Pharm Des 8:1765-1780

31. Chiriva-Internati M, Grizzi F, Pinkston J et al
(2006) Gamma-radiation upregulates MHC class I/II and ICAM-I molecules in multiple myeloma cell lines and primary tumors. In Vitro Cell Dev Biol Anim 42:89-95

32. Hareyama M, Imai K, Ban T et al (1988) Effect of radiation on the expression of carcinoembryonic antigen on the membranes of human gastric adenocarcinoma cells - immunological study using monoclonal antibodies. Nippon Igaku Hoshasen Gakkai Zasshi 48:1572-1574

33. Gaugler MH, Squiban C, van der Meeren A et al (1997) Late and persistent up-regulation of intercellular adhesion molecule-1 (ICAM-1) expression by ionizing radiation in human endothelial cells in vitro. Int J Radiat Biol 72:201-209

34. Garnett CT, Palena C, Chakraborty M et al (2004) Sublethal irradiation of human tumor cells modulates phenotype resulting in enhanced killing by cytotoxic T lymphocytes. Cancer Res 64:79857994

35. Kiewe P, Hasmüller S, Kahlert S et al (2006) Phase I trial of the trifunctional anti-HER2 $\mathrm{x}$ antiCD3 antibody ertumaxomab in metastatic breast cancer. Clin Cancer Res 12:3085-3091
36. Bokemeyer C, Heiss M, Gamperl H et al (2009) Safety of catumaxomab: cytokine-release-related symptoms as a possible predictive factor for efficacy in a pivotal phase II/III trial in malignant ascites. J Clin Oncol 27[Suppl]:Abstract 3036

37. Saito T, Berens ME, Welander CE (1986) Direct and indirect effects of human recombinant gamma-interferon on tumor cells in a clonogenic assay. Cancer Res 46:1142-1147

38. Gronau SS, Schmitt M, Thess B et al (2005) Trifunctional bispecific antibody-induced tumor cell lysis of squamous cell carcinomas of the upper aerodigestive tract. Head Neck 27: 376-382

39. Schmitt M, Schmitt A, Reinhardt P et al (2004) Opsonization with a trifunctional bispecific (alphaCD3 $\mathrm{x}$ alphaEpCAM) antibody results in efficient lysis in vitro and in vivo of EpCAM positive tumor cells by cytotoxic $\mathrm{T}$ lymphocytes. Int $\mathrm{J}$ Oncol 25:841-848

40. Hirschhaeuser F, Leidig T, Rodday B et al (2009) Test system for trifunctional antibodies in 3D MCTS culture. J Biomol Screen 14:980-990 